\title{
Incidence Rates of Autoimmune Diseases in European Healthcare Databases: A Contribution of the ADVANCE Project
}

\author{
Corinne Willame ${ }^{1}\left[\right.$ ] Caitlin Dodd ${ }^{1} \cdot{\text { Lieke van der } \mathrm{Aa}^{2} \cdot \text { Gino Picelli }^{3} \cdot \text { Hanne-Dorthe Emborg }}^{4} \cdot$ Johnny Kahlert $^{5}$. \\ Rosa Gini ${ }^{6}$. Consuelo Huerta ${ }^{7}$ Elisa Martín-Merino ${ }^{7} \cdot$ Chris McGee $^{8,9} \cdot$ Simon de Lusignan $^{8,9} \cdot$ Giuseppe Roberto $^{6}$. \\ Marco Villa $^{10} \cdot$ Daniel Weibel $^{11,12} \cdot$ Lina Titievsky $^{13} \cdot$ Miriam C. J. M. Sturkenboom $^{1,11,14}$
}

Accepted: 2 December 2020 / Published online: 19 January 2021

(c) The Author(s) 2021

\begin{abstract}
Introduction The public-private ADVANCE collaboration developed and tested a system to generate evidence on vaccine benefits and risks using European electronic healthcare databases. In the safety of vaccines, background incidence rates are key to allow proper monitoring and assessment. The goals of this study were to compute age-, sex-, and calendar-year stratified incidence rates of nine autoimmune diseases in seven European healthcare databases from four countries and to assess validity by comparing with published data.

Methods Event rates were calculated for the following outcomes: acute disseminated encephalomyelitis, Bell's palsy, Guillain-Barré syndrome, immune thrombocytopenia purpura, Kawasaki disease, optic neuritis, narcolepsy, systemic lupus erythematosus, and transverse myelitis. Cases were identified by diagnosis codes. Participating organizations/databases originated from Denmark, Italy, Spain, and the UK. The source population comprised all persons registered, with at least 1 year of data prior to the study start, or follow-up from birth. Stratified incidence rates were computed per database over the period 2003 to 2014.

Results Between 2003 and 2014, 148,947 incident cases of nine autoimmune diseases were identified. Crude incidence rates were highest for Bell's palsy [23.8/100,000 person-years (PYs), 95\% confidence interval (CI) 23.6-24.1] and lowest for Kawasaki disease (0.7/100,000 PYs, 95\% CI 0.6-0.7). Specific patterns were observed by sex, age, calendar time, and data sources. Rates were comparable with published estimates.

Conclusion A range of autoimmune events could be identified in the ADVANCE system. Estimation of rates indicated consistency across selected European healthcare databases, as well as consistency with US published data.
\end{abstract}

Supplementary Information The online version contains supplementary material available at https://doi.org/10.1007/s4026 4-020-01031-1.

Corinne Willame

c.willame@umcutrecht.nl

Extended author information available on the last page of the article

\section{Key Points}

In the safety of vaccines, background incidence rates are key to allow proper monitoring and assessment.

Between 2003 and 2014, 148,947 new cases of nine autoimmune diseases were identified in seven European healthcare databases from four countries.

Incidence rates were highest for Bell's palsy and lowest for Kawasaki disease. Specific patterns were observed by sex, age, calendar time, and data sources. 


\section{Introduction}

The Accelerated Development of VAccine beNefit-risk Collaboration in Europe (ADVANCE) was a public-private consortium launched by the Innovative Medicines Initiatives in 2013 to bring together stakeholders (i.e. regulators, academics, and vaccine manufacturers) actively involved in the postmarketing monitoring of benefits and risks (B/R) of vaccines [1]. The aim of the ADVANCE project was to build an efficient system to generate robust evidence on background rates and vaccine coverage and, ultimately, to rapidly assess the $\mathrm{B} / \mathrm{R}$ of vaccines using existing healthcare databases in Europe. ADVANCE has transitioned to the Vaccine Monitoring Collaboration for Europe that will implement the ecosystem [2]. In that context, several tools and methods have been developed to standardize ways of working among selected European healthcare databases. A description of the system and the methods/workflows can be found in the article by Sturkenboom et al. [1, 3].

With the entry of new vaccines to the market and their use on a large scale, rare adverse events not detected during clinical development phases may occur. Large sample sizes are required to rapidly evaluate suspected causal associations between rare adverse events such as autoimmune diseases and vaccines in a real-world setting. Preparedness to investigate safety signals and safety concerns is a necessary requirement of vaccination programs stipulated in the Vaccine Safety Blueprint [4]. Based on a stakeholder analysis in Europe, background rates are important from a regulatory, manufacturer, and public health perspective [1]. Because of the mode of action of vaccines and the fact that adjuvants, which stimulate immune response, may be used, autoimmune diseases are often events of interest to monitor and investigate. This is especially relevant considering that they have age-related patterns of onset that may coincide with age at vaccination. Moreover, autoimmune diseases are rare and because of the possible impact of environmental factors on their occurrence $[5,6]$, there is a constant need to generate up-to-date background incidence rates (IRs). As part of being prepared to respond to signals, background rates are a crucial source of information in the assessment of suspected cases, especially during mass vaccination campaigns [7] or for continuous safety monitoring of vaccines in a growing recipient population [8].

As part of the database characterization efforts of the ADVANCE project, we estimated background IRs of nine autoimmune diseases. We described and tried to explain heterogeneity among sources of data (e.g. hospital-based outcomes and/or primary care-based), and compared them with external published data [9].

\section{Methods}

\subsection{Setting}

The ADVANCE project had access to 20 different data sources, seven of which could be used in this assessment, representing four countries-Denmark, Spain, Italy, and the UK (Table 1). Detailed descriptions of these databases can be found in the electronic supplementary file.

All participating data sources extracted study data into a common data model (CDM). As described by Sturkenboom et al. [10], the CDM comprises three data files-population, events and vaccinations.

\subsection{Population}

The source population comprised all persons registered with at least 1 year of data prior to the start of the study period or follow-up from birth. Data for all individuals recorded in each database from the start of follow-up (defined as birth or first data availability, whichever was latest) until the end of follow-up (defined as the date at last data retrieval, leaving the database, the date of first event, or death, whichever date was earliest), were used to define the follow-up for database characterization. The only eligibility criteria were that the date of birth, start and end follow-up dates, and sex needed to be available. The study start date varied between databases, depending on when the database collection started, and ended in 2017 for all databases. Data access providers (DAPs) created a population file in the format of the CDM including patient identifier, start of follow-up date, end of follow-up date, birth date, and sex.

\subsection{Events}

The autoimmune diseases of interest were acute disseminated encephalomyelitis (ADEM), Bell's palsy, Guillain-Barré syndrome (GBS), immune thrombocytopenia purpura (ITP), Kawasaki disease, optic neuritis, narcolepsy, systemic lupus erythematosus (SLE), and transverse myelitis. The outcomes were defined using definitions from the Brighton Collaboration and learned societies, the World Health Organization, or the European Centre for Disease Prevention and Control. The case definitions were mapped to an initial list of the International Classification of Diseases, Ninth Revision, Clinical Modification (ICD-9-CM), and ICD Tenth Revision (ICD-10), Read, and the International Classification of Primary Care (ICPC) codes using the ADVANCE Code mapper tool [11]. DAPs for each database were asked to modify and verify the proposed codes based on local coding habits and prior experience. Each DAP 


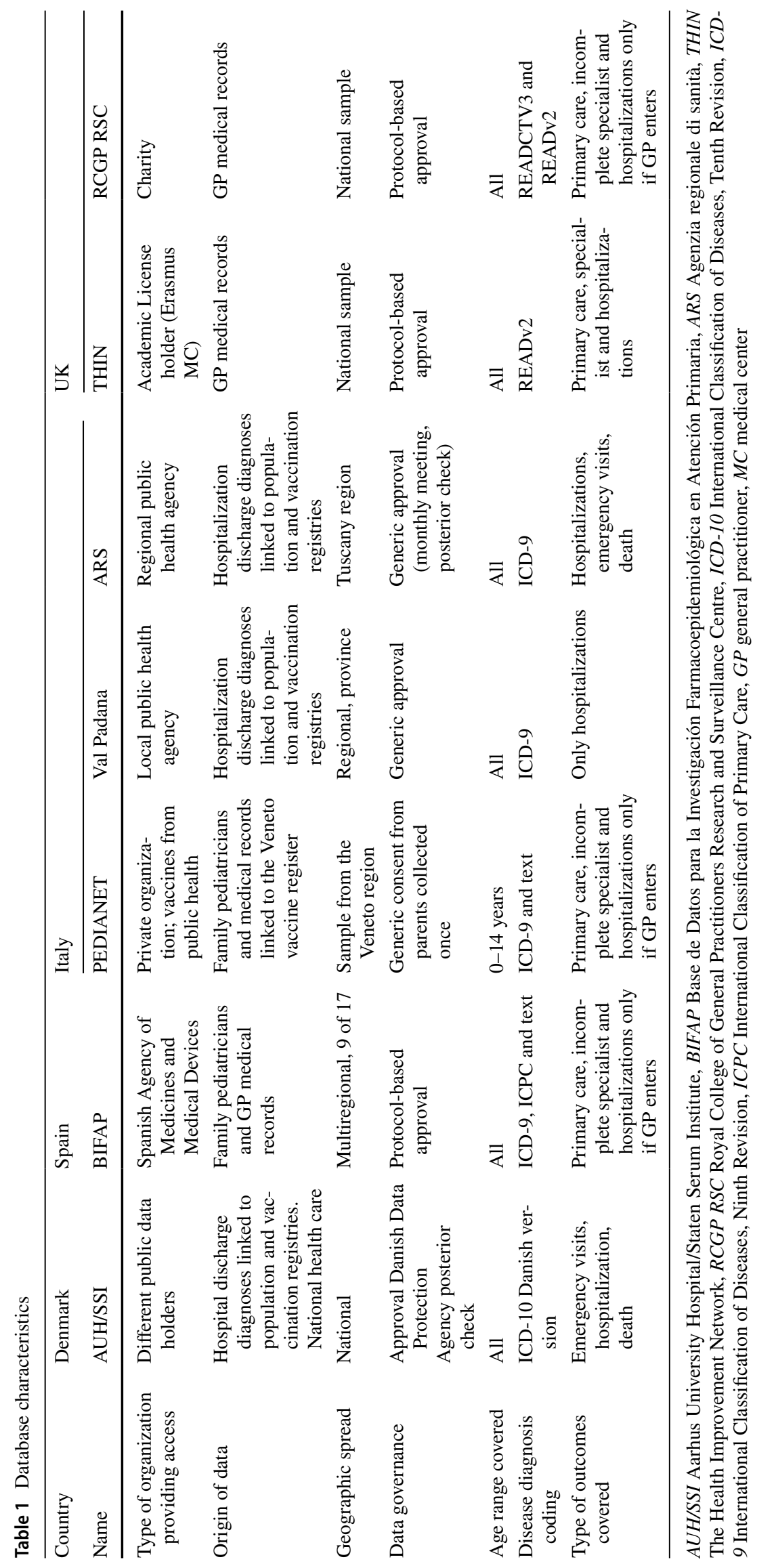


extracted the final list of codes for the specific events in their local terminology and transformed the data into the event file of the CDM containing the following fields: patient identifier, event type, date, original code (ICD-9/10, Read, ICPC, or text). The event file was linked to the population file to calculate event IRs and to assess whether these rates were as expected by benchmarking rates within the data source, between data sources, and against published data. This assessment allowed us to demonstrate the appropriateness of the data processing steps used. The code list for each outcome of interest is available in electronic supplementary Table S1. The ITP condition was defined according to narrow and broad concepts. Details on the harmonization process for data extraction are described elsewhere [10].

\subsection{Data Management and Analyses}

The DAPs extracted data from their database using the local data format and software, which were transformed into the ADVANCE CDM (CSV format). We used Jerboa data processing software, which is JAVA-based, for event code counting and incidence calculations. The Jerboa software has been used for multiple studies and is freely available. The script and instructions were sent to the DAPs, who ran the script against their input files, and the outputs were sent through a secure file transfer protocol (File Zilla or HighTail) to a private remote research environment (PRRE) [10].

The event characterization included code counts by type of event and database, and event IRs in the population by calendar year, sex, and age. Age was categorized per year until 17 years, from 18-24 years, and then in 5-year categories. We subsequently categorized age in $0-1,2-4,5-14,15-24$, $25-64$, and $\geq 65$ years for description, as this coincides with age of routine vaccination in general and because this categorization was compatible with the Post-licensure Rapid Immunization Safety Monitoring programme (PRISM) [9] US database age categories, allowing for age-specific comparisons of IRs between the US and European networks. For the incidence estimates calculated with Jerboa, there was a 1-year run-in period for individuals aged 6 months onward; individuals with an entry date within 6 months of birth started their follow-up at birth. Events recorded in the 1-year run-in prior to the start of follow-up were not considered and only first events recorded after the run-in period were considered to be incident. To have a comparable period of calendar time across databases, IRs were limited to the calendar years 2003-2014. Healthcare databases were classified according to the type of data sources: general practitioner databases including Base de Datos para la Investigación Farmacoepidemiológica en Atención Primaria (BIFAP), The Health Improvement Network (THIN), Royal College of General Practitioners (RCGP) Research and Surveillance Centre (RSC), and Pedianet and hospitalization record linkage databases, including Aarhus University Hospital (AUH)/Staten Serum Institute (SSI), Agenzia regionale di sanità (ARS), and Val Padana. We calculated crude IRs as the number of incident events within the follow-up period divided by the total person-time at risk and $95 \%$ confidence intervals (CIs) using the exact method for each event. IRs were expressed per 100,000 person-years (PYs). We also computed yearly pooled IRs for each autoimmune disease to compare the type of data sources (general practitioners vs. hospitalization record linkage) by using a random-effects model (Der Simonian-Laird method). Higgins $I^{2}$ statistics were measured to determine heterogeneity between the type of data sources. Upon higher rates of narcolepsy observed in AUH/SSI, we conducted a post hoc analysis to estimate age-stratified IRs of narcolepsy in Denmark over the study period. Data handling and computation of rates were performed in SAS 9.4 (SAS Institute Inc., Cary, NC, USA) and meta-analyses were conducted in Stata v14.0 (StataCorp LLC, College Station, TX, USA.

\section{Results}

Over the period 2003 to 2014, the total person-time of follow-up was more than 233 million PYs for the seven European healthcare databases. The largest contributions in follow-up were from AUH/SSI databases (30.9\%), THIN (27.0\%), and ARS (20.0\%) (Table 2). The population aged between 15 and 64 years has most of the person-time represented in each database, except for Pedianet, which only captures the pediatric population. Between 2003 and 2014, there were 148,947 incident cases of nine predefined autoimmune diseases. Of the nine individual autoimmune diseases, the crude IR of Bell's palsy was the highest $(23.8 / 100,000$ PYs, 95\% CI 23.6-24.1), followed by ITP broad definition (21.7/100,000 PYs, 95\% CI 21.6-22.0), SLE (5.3/100,000 PYs, 95\% CI 5.2-5.4), ADEM (5.3/100,000 PYs, 95\% CI 5.2-5.3), ITP narrow definition (3.8/100,000 PYs, 95\% CI 3.7-3.9), optic neuritis (3.4/100,000 PYs, 95\% CI 3.3-3.5), GBS (2.1/100,000 PYs, 95\% CI 2.0-2.1), narcolepsy (1.1/100,000 PYs, 95\% CI 1.0-1.1), transverse myelitis (1.0/100,000 PYs, 95\% CI 0.9-1.0), and Kawasaki disease (0.7/100,000 PYs, 95\% CIs 0.6-0.7). The sex-specific crude IRs of several autoimmune diseases were higher in females than in males (Table 3), and the most pronounced was SLE, with an IR of 8.5/100,000 PYs in females and 2.1/100,000 PYs in males. For each database, age- and sex-specific crude IRs are presented in electronic supplementary Table S2.

\subsection{Age-Stratified Incidence Rates Per Database}

Overall and age-stratified IRs are presented in Table 4. We observed that the age patterns differ across different 
Table 2 Follow-up duration and number of autoimmune events for each database over the period 2003-2014

\begin{tabular}{|c|c|c|c|c|c|c|c|}
\hline & \multirow{2}{*}{$\begin{array}{l}\text { Denmark } \\
\text { AUH/SSI }\end{array}$} & \multicolumn{3}{|l|}{ Italy } & \multirow{2}{*}{$\begin{array}{l}\text { Spain } \\
\text { BIFAP }\end{array}$} & \multicolumn{2}{|l|}{ UK } \\
\hline & & ARS & Val Padana & Pedianet & & RCGP RSC & THIN \\
\hline \multicolumn{8}{|c|}{ Person-time (in years) per age groups and databases } \\
\hline Overall & $71,963,997$ & $46,690,197$ & $4,429,415$ & 414,725 & $29,654,858$ & $16,845,082$ & $63,107,306$ \\
\hline $0-1$ years & $1,630,983$ & 784,016 & 78,040 & 82,862 & 696,022 & 438,305 & $1,572,725$ \\
\hline $2-4$ years & $2,495,269$ & $1,152,313$ & 116,835 & 110,105 & 888,063 & 571,917 & $2,127,038$ \\
\hline $5-14$ years & $9,224,436$ & $3,910,548$ & 404,509 & 221,758 & $2,721,747$ & $1,916,302$ & $7,140,915$ \\
\hline $15-24$ years & $10,413,928$ & $3,951,513$ & 382,907 & - & $4,936,428$ & $1,945,611$ & $6,908,561$ \\
\hline $25-44$ years & $19,350,418$ & $13,002,241$ & $1,177,745$ & - & $9,343,807$ & $4,601,269$ & $17,333,183$ \\
\hline 45-64 years & $17,953,083$ & $12,977,224$ & $1,247,063$ & - & $6,829,082$ & $4,454,574$ & $17,064,552$ \\
\hline $65+$ years & $10,895,877$ & $10,912,341$ & $1,022,316$ & - & $4,239,709$ & $2,917,103$ & $10,960,332$ \\
\hline \multicolumn{8}{|c|}{ Total number of incident event databases for each autoimmune disease } \\
\hline \multicolumn{8}{|l|}{ Autoimmune diseases } \\
\hline ADEM & 3866 & 5521 & 527 & 6 & 619 & 353 & 601 \\
\hline Bell's palsy & 14,087 & 2758 & 130 & 24 & 12,542 & 4194 & 18,398 \\
\hline GBS & 1711 & 1085 & 109 & $<5$ & 321 & 257 & 1021 \\
\hline ITP (broad definition) & 10,020 & 8970 & 474 & 11 & 14,796 & 3447 & 9923 \\
\hline ITP (narrow definition) & 3775 & 872 & 63 & 7 & 484 & 639 & 2536 \\
\hline Kawasaki disease & 412 & 420 & 12 & 30 & 47 & 123 & 407 \\
\hline Narcolepsy & 1333 & 144 & 7 & $<5$ & 201 & 132 & 549 \\
\hline Optic neuritis & 2982 & 1,048 & 72 & $<5$ & 694 & 533 & 2163 \\
\hline SLE & 3526 & 1,438 & 151 & $<5$ & 1985 & 1078 & 3477 \\
\hline Transverse myelitis & 678 & 144 & 12 & $<5$ & $<5$ & 213 & 783 \\
\hline
\end{tabular}

AUH/SSI Aarhus University Hospital/Staten Serum Institute, BIFAP Base de Datos para la Investigación Farmacoepidemiológica en Atención Primaria, ARS Agenzia regionale di sanità, THIN The Health Improvement Network, RCGP RSC Royal College of General Practitioners Research and Surveillance Centre, ADEM acute disseminated encephalomyelitis, GBS Guillain-Barré syndrome, ITP immune thrombocytopenia purpura, $S L E$ systemic lupus erythematosus

Table 3 Crude incidence rates (/100,000 PYs) per sex for each autoimmune disease

\begin{tabular}{lcc}
\hline Autoimmune diseases & \multicolumn{2}{l}{ IR $(95 \%$ CI) per 100,000 PYs } \\
\cline { 2 - 3 } & Female & Male \\
\hline ADEM & $6.14(6.00-6.29)$ & $4.31(4.19-4.44)$ \\
Bell's palsy & $23.82(23.54-24.11)$ & $23.86(23.57-24.15)$ \\
GBS & $1.74(1.66-1.82)$ & $2.39(2.30-2.48)$ \\
ITP (broad definition) & $20.47(20.20-20.73)$ & $23.11(22.83-23.40)$ \\
ITP (narrow definition) & $3.95(3.84-4.07)$ & $3.69(3.57-3.80)$ \\
Kawasaki disease & $0.52(0.47-0.56)$ & $0.81(0.76-0.87)$ \\
Narcolepsy & $1.12(1.06-1.19)$ & $1.04(0.98-1.10)$ \\
Optic neuritis & $4.42(4.29-4.54)$ & $2.39(2.29-2.48)$ \\
SLE & $8.47(8.30-8.65)$ & $2.05(1.97-2.14)$ \\
Transverse myelitis & $1.10(1.03-1.17)$ & $0.83(0.77-0.89)$ \\
\hline
\end{tabular}

$A D E M$ acute disseminated encephalomyelitis, GBS Guillain-Barré syndrome, ITP immune thrombocytopenia purpura, SLE systemic lupus erythematosus, $P Y$ person-years, $I R$ incidence rate, $C I$ confidence interval

autoimmune diseases: IRs increased with increasing age for Bell's palsy, GBS, and SLE. The narrow definition of ITP shows the highest rates in the $0-4$ years age group. This rate decreased in children aged between 5 and 24 years, and increased by age from the age of 25 years. A similar pattern with a higher magnitude of rates was observed using the ITP broad definition. In the elderly ( $65+$ years) IRs ranged between 22 and 64/100,000 PYs, except in BIFAP, where IRs peaked at 130/100,000 PYs. IRs for narcolepsy were low ( $\leq 1 / 100,000$ PYs), but slightly higher rates were observed in the Danish database. In Denmark, the IR for narcolepsy was as high as 3.1/100,000 PYs in the 15-24 age group. A specific analysis of this age group per calendar year in the AUH/SSI database showed that IRs increased at the beginning of the study period and tended to level out during the period 2008-2012, potentially followed by a slight increase towards the end of the study period (Fig. 1). The pattern of IRs for optic neuritis was similar across databases, increasing by age and peaking in the 25-44 years age group, except in the BIFAP database, where a constant increase by age was observed. Although no clear pattern was observed for ADEM, IRs peaked in the 25-44 years age group in both 


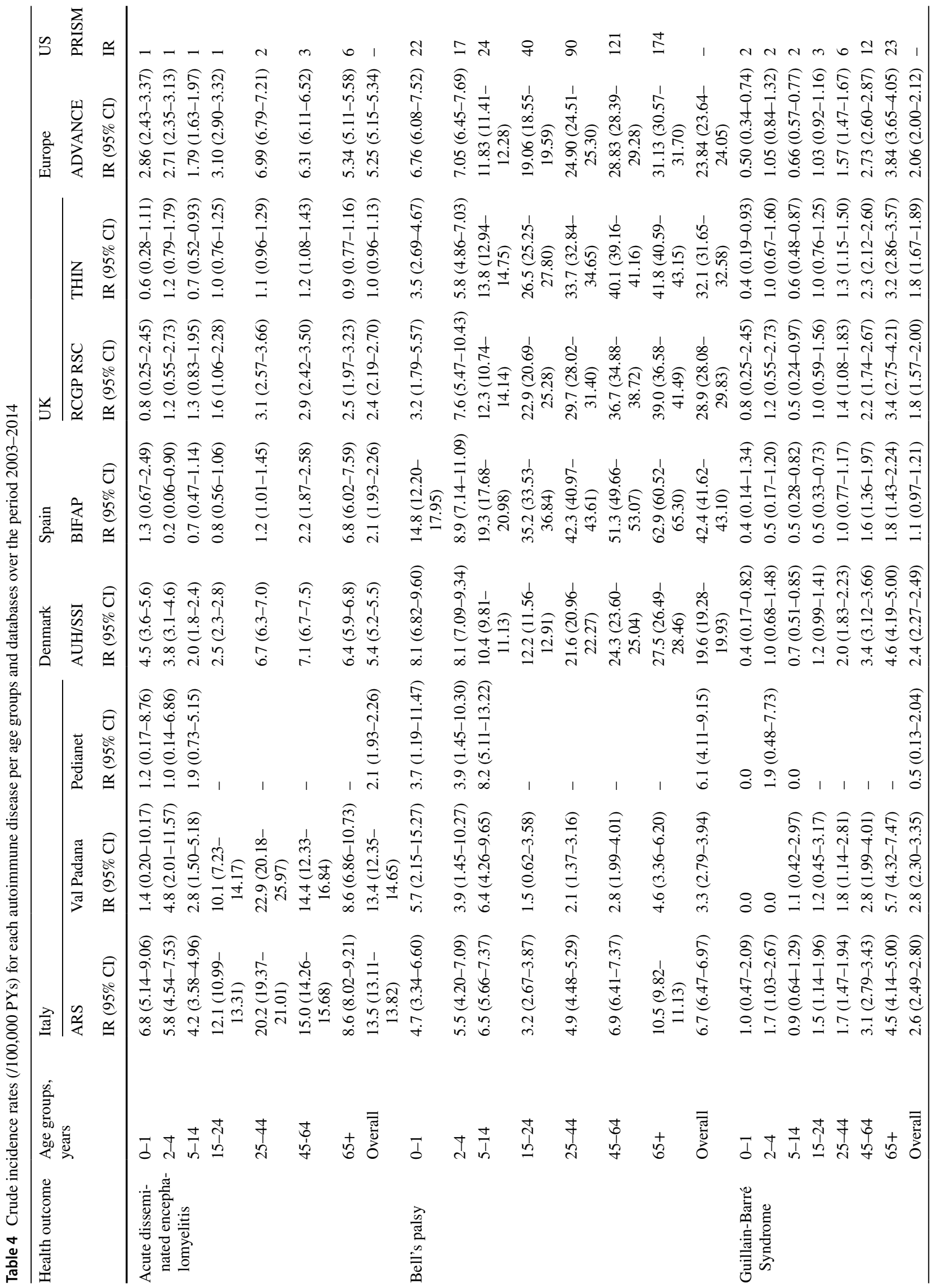




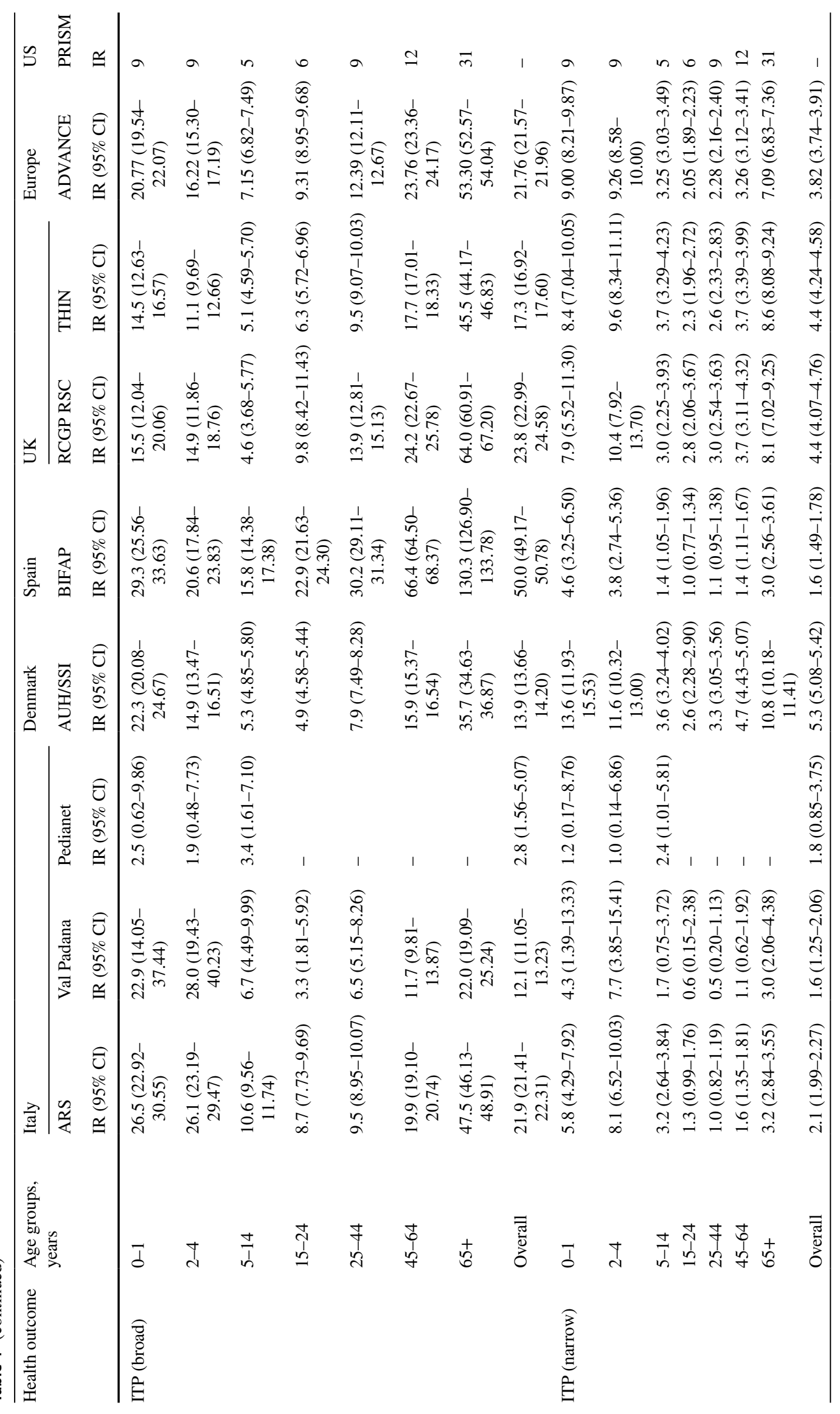




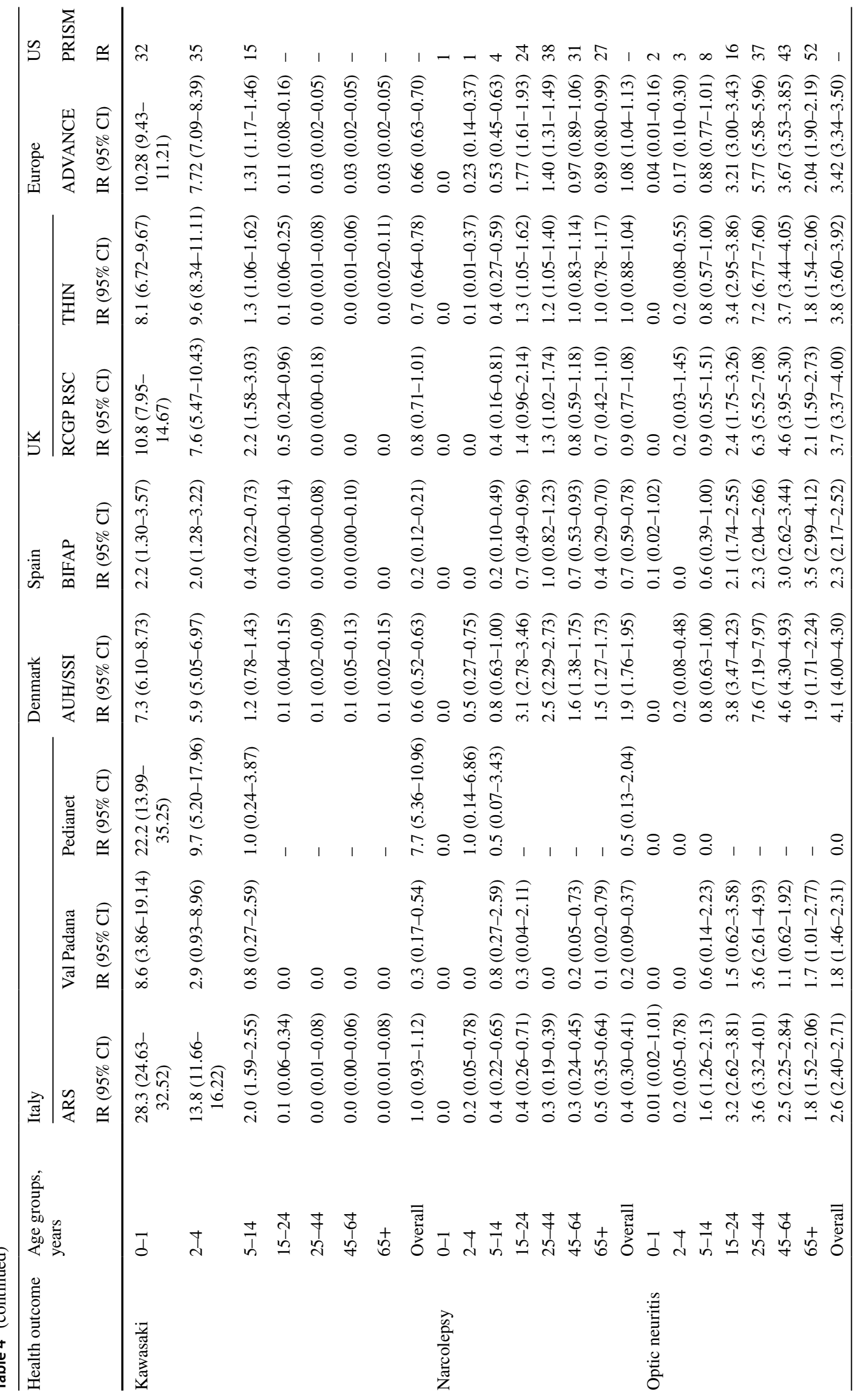




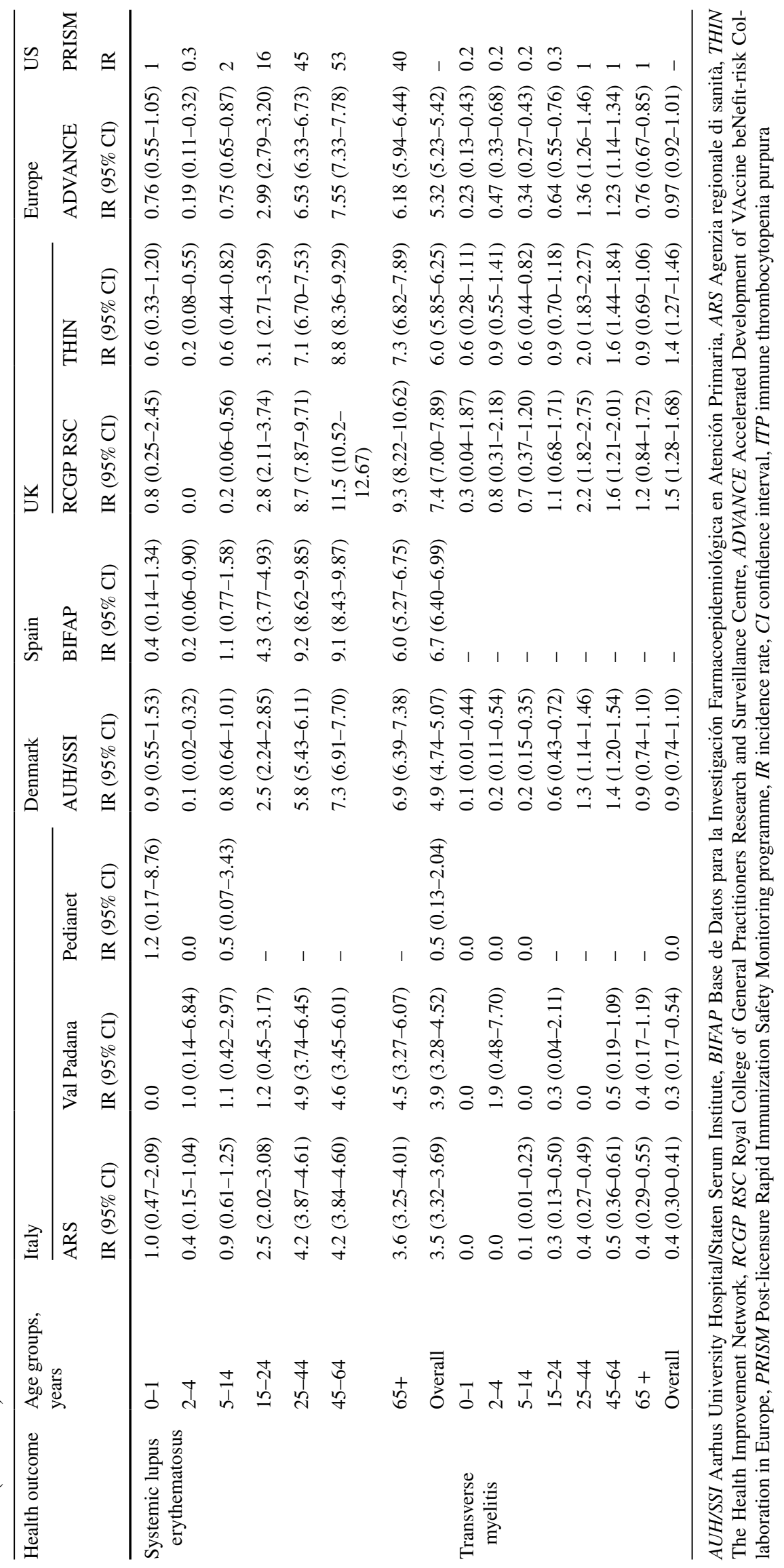




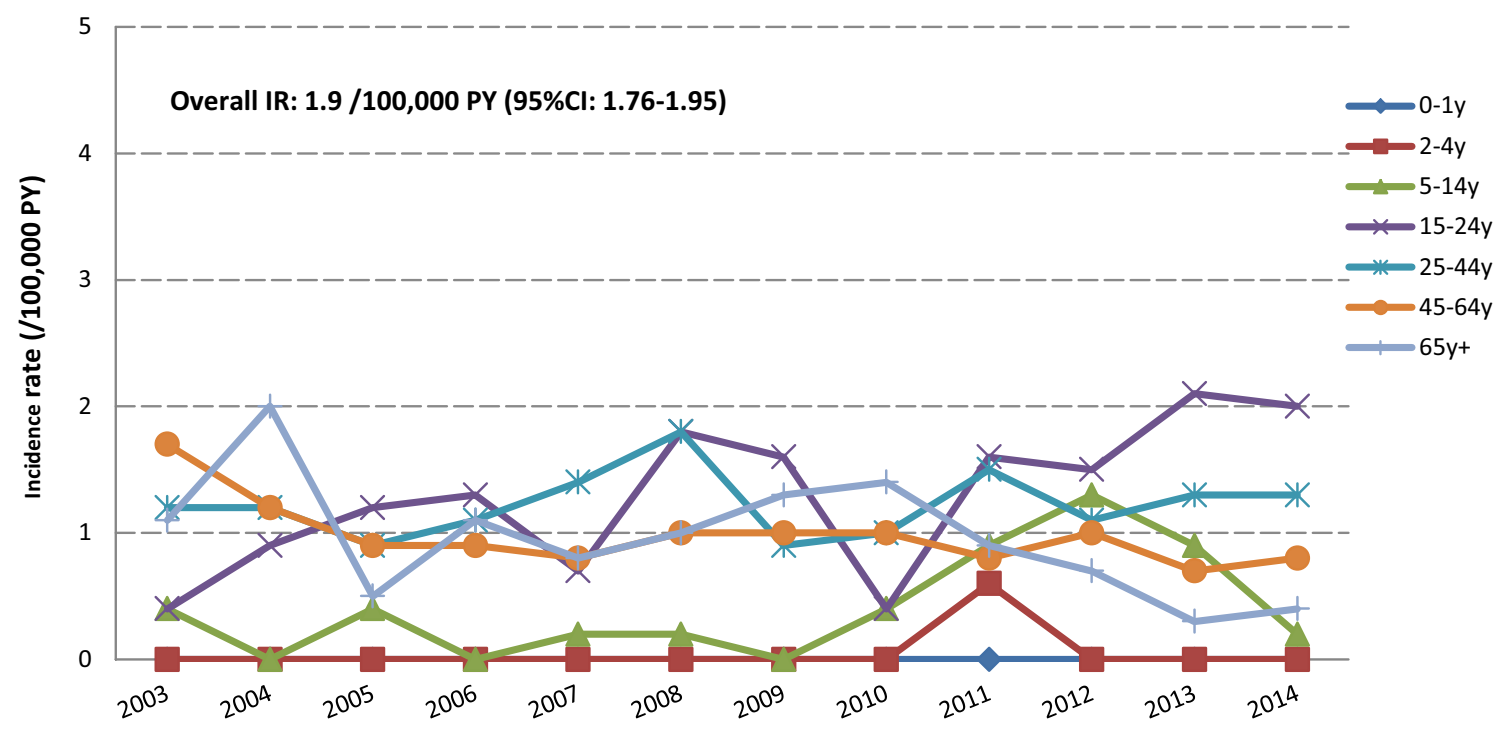

Fig. 1 Incidence rates for narcolepsy in the AUH/SSI database, per age group and calendar year. $I R$ incidence rate, $P Y$ person-years, $C I$ confidence interval, AUH/SSI Aarhus University Hospital/Staten Serum Institute

the record linkage Italian databases (ARS and ASCLR). The pattern of IRs for Kawasaki disease was similar across databases, with most of the events occurring before the age of 14 years. IRs for transverse myelitis varied from 0.0 to 2.2/100,000 PYs; no events were reported in the BIFAP and Pedianet databases.

\subsection{Incidence Rates Over Calendar Years According to the Type of Data Sources}

Yearly pooled IRs of autoimmune diseases were stable over time but differed by type of data source for some diseases (electronic supplementary Fig. S1). IRs of ADEM and GBS were higher in hospital-based record linkage databases than in primary care databases. On the contrary, IRs of Bell's palsy, ITP narrow, Kawasaki, SLE, and transverse myelitis were higher in primary care databases.

\section{Discussion}

In this study, we estimated age-, sex-, and calendar timespecific background rates of nine autoimmune diseases of interest for vaccine safety assessment from seven European electronic healthcare databases. We demonstrated that the ADVANCE system could detect age-specific patterns and differences in IRs by the origin of information (e.g. hospital or general practioners) as well as sex. IRs were fairly stable over time for each disease, showing that identification or recording was not modified during the study period. The age-dependent patterns are important to know for the calculation of observed versus expected cases, as some of the age categories in which rates increase coincide with the age of vaccination. The ADVANCE tools allowed for rapid estimation of the rates by age, calendar time, and sex. Overall, IRs from the ADVANCE system were of a lower magnitude than rates generated through the US PRISM system, which covers claims-based diagnoses from outpatients, emergency units, and hospitalization. Age-specific patterns were similar for most of the autoimmune diseases, i.e. ADEM, Bell's palsy, GBS, narcolepsy, optic neuritis, SLE, and transverse myelitis. IRs for ITP narrow definition matched rates from the US PRISM system more closely than those for the ITP broad definition. For both systems, PRISM and ADVANCE, we observed the highest rates for Kawasaki disease in children $<4$ years of age. The female predominance in SLE is also consistent with recent published literature [12], with the female:male ratio for SLE ranging from 4:1 to 9:1, which is aligned with our observation (4:1). In all databases, IRs for optic neuritis peaked between the ages of 25 and 44 years, decreasing thereafter, except in BIFAP, where we observed a constant increase by age. Estimates of the incidence of optic neuritis have been published from Barcelona [13], another region in Spain for which data are not captured in BIFAP. The data from Barcelona also confirmed the peak of IRs for optic neuritis in the 20-40 years age group over the period from 2008 to 2012. The reason for this variation in rates for optic neuritis between BIFAP and the other databases in ADVANCE is unknown. The ICPC code that was used is specific for optic neuritis, but this code may be used in clinical practice to code suspected conditions as a reason for referral to specialists allowing for testing, diagnosis and confirmation. IRs for narcolepsy were low and stable over time $\leq 1 / 100,000$ PYs, except in Denmark, where the rate 
of narcolepsy diagnosis was slightly elevated and showed periods with increases in persons between 15 and 24 years of age. However, an increase in the incidence of narcolepsy in Denmark was previously observed, and happened prior to the administration of the influenza A(H1N1)pdm09 pandemic vaccine, which has been associated with increases in the IR of narcolepsy in Finland, Norway, Ireland, and Sweden $[14,15]$, but not in countries with low vaccine coverage [16].

Comparisons of our data with the US PRISM system showed similar age patterns in IRs [10]. Rates from PRISM, which is based on US claims data, were generally higher than the rates we observed in Europe. This may have several causes: coverage of outpatient specialist diagnoses, inclusion of prevalent cases, generally higher disease rates, or careseeking behavior. With regard to European published data, high similarities in rate patterns have been observed for most of the diseases, such as Bell's palsy or GBS [7], Kawasaki disease $[17,18]$ or narcolepsy [16]. Nevertheless, no direct comparison could be made for several reasons: no overlapping in age strata, ascertainment methods used, diverse sources of data, and their geographical location. Overall, this benchmark provides reassurance about external validity.

We demonstrated that all the participating databases provide crude rates consistent with expectations. However, our pooled crude rates should be interpreted with caution because they were not adjusted for any relevant covariates, nor were they weighted by the data sources with the largest person-time contribution, and should only be used in the context of each individual DAP's results. Misclassification of incidence as prevalence may occur due to differences in health care provision, as some diagnoses are made in primary care whereas others may lead to hospitalization, and most of the databases do not capture all health care sites. Our analysis by type of data source highlights the specific process of diagnosis of autoimmune diseases. The quantification of these differences is important to realize when designing a specific study, and may profit from the component strategy introduced in the ADVANCE project for this purpose [19]. Background rates of adverse events of special interest following immunization are always needed to conduct observed/expected analyses [7, 20], to understand burden of disease of adverse events [21], or in cost-evaluation of vaccine implementation [22].

\section{Conclusion}

This study demonstrated that the European ADVANCE system can identify specific autoimmune events, that age-, sex- and time-specific rates can be generated based on available tools, and that the IRs are mostly consistent across selected European healthcare databases. Some variations were observed according to the type of care that is captured in the data sources.

Acknowledgements The authors wish to thank Lara Tramontan for her support in this project and her critical review of the manuscript. They would also like to express their gratitude for the excellent collaboration of the primary care practitioners and pediatricians, and the support of the regional authorities participating in this project.

\section{Declarations}

Funding The Innovative Medicines Initiative Joint Undertaking funded this project under ADVANCE Grant agreement number 115557, resources of which were composed of a financial contribution from the European Union's Seventh Framework Programme (FP7/2007-2013) and in kind contributions from European Federation of Pharmaceutical Industries and Associations (EFPIA) member companies.

Conflict of interest At the time of the study and manuscript development, Corinne Willame worked part time with the GlaxoSmithKline (GSK) group of companies and declares not to receive any financial support from the GSK group of companies for the submitted work. At the time of the study and manuscript development, Lina Titievsky was a Pfizer employee. Rosa Gini is employed by ARS, a public health agency that conducts or participates in pharmacoepidemiology studies. The budget of ARS is partially sustained by such studies. Simon de Lusignan has been a member of Advisory Boards for Sanofi and Seqirus, and has received funding for vaccine-related researches from GSK, Seqirus, and Takeda. Daniel Weibel has been a consultant for GSK's malaria vaccine program. Miriam Sturkenboom is principal investigator of post-authorization safety studies requested by the European Medicines Agency, for Novartis, none of which were related to the topic described. Caitlin Dodd, Lieke van der Aa, Gino Picelli, Hanne-Dorthe Emborg, Johnny Kahlert, Consuelo Huerta, Elisa Martín-Merino, Chris McGee, Giuseppe Roberto, and Marco Villa declare they have no conflicts of interest.

Ethical approval For this type of study, governance approval is mandatory to access data sources.

Consent to participate Not applicable.

Consent for publication Not applicable.

Code availability Not applicable.

Availability of data and material Data are available from the DAPs of each of the participating data sources for researchers who meet the criteria to access confidential data. The minimal underlying dataset for replication would require interested researchers to contact each individual listed for access to their data.

Authors' contributions All authors contributed equally to the work submitted.

Open Access This article is licensed under a Creative Commons Attribution-NonCommercial 4.0 International License, which permits any non-commercial use, sharing, adaptation, distribution and reproduction in any medium or format, as long as you give appropriate credit to the original author(s) and the source, provide a link to the Creative Commons licence, and indicate if changes were made. The images or other third party material in this article are included in the article's Creative Commons licence, unless indicated otherwise in a credit line to the 
material. If material is not included in the article's Creative Commons licence and your intended use is not permitted by statutory regulation or exceeds the permitted use, you will need to obtain permission directly from the copyright holder. To view a copy of this licence, visit http://creativecommons.org/licenses/by-nc/4.0/.

\section{References}

1. Sturkenboom M, Bahri P, Chiucchiuini A, Grove Krause T, Hahne S, Khromava A, et al. Why we need more collaboration in Europe to enhance post-marketing surveillance of vaccines. Vaccine. 2019. https://doi.org/10.1016/j.vaccine.2019.07.081.

2. Vaccine monitoring Collaboration for Europe. VAC4EU (2019). Available on: https://vac4eu.org/. Accessed on 18 Dec 2020.

3. Sturkenboom M, van der Aa L, Bollaerts K, Emborg HD, Ferreira G, Gino R, et al. The ADVANCE distributed network system for evidence generation on vaccines coverage, benefits and risks based on electronic health care data. Vaccine. 2020; Suppl 38:B76-B83.

4. Giesecke J, Kramarz P, Kokki M, for the ADVANCE project team. Blueprint of a framework to rapidly provide scientific evidence on post-marketing vaccination benefits and risks for informed decisions. WP7-Implementability analysis. V2.0. ADVANCE Consortium; 2018. Available on: https://vac4eu.org/wp-content/ uploads/2019/02/D77Blueprint.pdf. Accessed on 18 Dec 2020.

5. Shukla S, Singh G, Ahmad S, Pant P. Infections, genetic and environmental factors in pathogenesis of autoimmune thyroid diseases. Microb Pathog. 2018;116:279-88.

6. Jorg S, Grohme D, Erzler M, Binsfeld M, Haghikia A, Muller D, et al. Environmental factors in autoimmue diseases and their role in multiple sclerosis. Cell Mol Life Sci. 2016;73:4611-22.

7. Black S, Eskola J, Siegrist CA, Halsey N, MacDonald N, Law B, et al. Importance of background rates of disease in assessment of vaccine safety during mass immunization with pandemic H1N1 influenza vaccines. Lancet. 2009;374:2115-22.

8. Kurz X, Domergue F, Slattery J, Segec A, Szmigiel A, HidalgoSimon A. Safety monitoring of influenza A/H1N1 pandemic vaccines in EudraVigilance. Vaccine. 2011;29:4378-87.

9. Baker M, Nguyen M, Cole D, Lee G, Lieu T. Post-licensure rapid immunization safety monitoring program (PRSIM) data characterization. Vaccine. 2013;31S:K98-112.

10. Sturkenboom M, Braeye T, van der Aa L, Becker B, Berensci K, Danieli $\mathrm{G}$, et al. ADVANCE database characterization and fit for purpose assessment for multi-country studies on the coverage, benefits and risks for pertussis vaccinations. Vaccine. 2019; 38 Suppl 2:B8-21.
11. Becker B, Avillach P, Romio S, van Mulligen E, Weibel D, Sturkenboom M, Kors J, for the ADVANCE consortium. CodeMapper: semiautomatic coding of case definitions. A contribution from the ADVANCE project. Pharmacoepidemiol Drug Saf. 2017;26:998-1005.

12. Ji J, Sundquist J, Sundquist K. Gender-specific incidence of autoimmune diseases from national registers. J Autoimmun. 2016;69:102-6.

13. Martinez-Lapiscina E, Fraga-Pumar E, Pastor X, Gomez M, Conesa A, Lozano-Rubi R, et al. Is the incidence of optic neuritis rising? Evidence from an epidemiological study in Barcelona (Spain), 2008-2012. J Neurol. 2014;261:759-67.

14. Wijnans L, Lecomte C, de Vries C, Weibel D, Sammon C, Hviid A, et al. The incidence of narcolepsy in Europe: before, during, and after the influenza $\mathrm{A}(\mathrm{H} 1 \mathrm{~N} 1)$ pdm09 pandemic and vaccination campaigns. Vaccine. 2013;31:1246-54.

15. O'Flanagran D, Barret AS, Foley M, Cotter S, Bonner C, Crowe $\mathrm{C}$, et al. Investigation of an association between onset of narcolepsy and vaccination with pandemic influenza vaccine, Ireland April 2009-December 2010. Euro Surveill. 2014;19:17.

16. Dodd C, de Ridder M, Huang WT, Weibel D, Giner-Soriano M, Perez-Vilar S, et al. Incidence rates of narcolepsy diagnoses in Taiwan, Canada, and Europe: the use of statistical simulation to evaluate methods for the rapid assessment of potential safety issues on a population level in the SOMNIA study. PLoS ONE. 2018;10(13):e0204799.

17. Hall G, Tulloh L, Tilloh R. Kawasaki disease incidence in children and adolescents: an observational study in primary care. Br J Gen Pract. 2016;66(645):e271.

18. Cimaz R, Fanti E, Mauro A, Voller F, Rusconi F. Epidemiology of Kawasaki disease in Italy: surveillance from national hospitalization records. Eur J Pediatr. 2017;176:1061-5.

19. Gini R, Dodd CN, Bollaerts K, Bartolini C, Roberto G, Huerta-Alvarez $\mathrm{C}$, et al. Quantifying outcome misclassification in multi-database studies: the case study of pertussis in the ADVANCE project. Vaccine. 2019. https://doi.org/10.1016/j.vacci ne.2019.07.045.

20. Mahaux O, Bauchau V, Van Holle L. Pharmacoepidemiological considerations in observed-to-expected analyses for vaccines. Pharmacoepidemiol Drug Saf. 2016;25(2):215-22.

21. Mac Donald S, Nijsten D, Bollaerts K, Bauwens J, Praet N, van der Sande M, et al. Methodology for computing the burden of disease of adverse events following immunization. Pharmacoepidemiol Drug Saf. 2018;27(7):724-30.

22. Van Hoek A, Choi YH, Trotter C, Miller E, Jit M. The cost-effectiveness of a 13-valent pneumococcal conjugate vaccination for infants in England. Vaccine. 2012;30:7205-13.

\section{Authors and Affiliations}

\section{Corinne Willame ${ }^{1}$ (D) Caitlin Dodd ${ }^{1} \cdot{\text { Lieke van der } \mathrm{Aa}^{2} \cdot \text { Gino Picelli }^{3} \cdot \text { Hanne-Dorthe Emborg }}^{4} \cdot$ Johnny Kahlert $^{5}$. Rosa Gini $^{6}$. Consuelo Huerta ${ }^{7}$. Elisa Martín-Merino ${ }^{7}$. Chris McGee ${ }^{8,9} \cdot$ Simon de Lusignan $^{8,9}$. Giuseppe Roberto ${ }^{6}$. Marco Villa ${ }^{10} \cdot$ Daniel Weibel $^{11,12} \cdot$ Lina Titievsky $^{13} \cdot$ Miriam C. J. M. Sturkenboom $^{1,11,14}$}

1 Julius Global Health, University Medical Center Utrecht, Heidelberglaan 100, Utrecht, The Netherlands

2 Sciensano, Rue Juliette Wytsmanstraat 14, 1050 Brussels, Belgium
3 Epidemiological Information for Clinical Research from an Italian Network of Family Paediatricians (Pedianet), Padua, Italy

4 Department of Infectious Disease Epidemiology and Prevention, Statens Serum Institut, Artillerivej 5, 2300 Copenhagen, Denmark 
5 Aarhus University Hospital, Olof Palmes Alle 43-45, 8200 Aarhus, Denmark

6 Agenzia regionale di sanità della Toscana, Osservatorio di epidemiologia, Florence, Italy

7 Spanish Agency of Medicines and Medical Devices-AEMPS, Madrid, Spain

8 University of Surrey, Oxford, UK

9 Royal College of General Practitioners, Research and Surveillance Centre, 30 Euston Square, London, UK
10 Ospedale Val Padana, Cremona, Italy

11 VACCINE.GRID, Basel, Switzerland

12 Erasmus University Medical Center, PO Box 2014, 3000 CA Rotterdam, The Netherlands

13 Pfizer, New York, NY, USA

14 P-95, Koning Leopold III laan 1 3001, Heverlee, Leuven, Belgium 